\title{
Radio Coverage Analysis of Anchor Nodes for the Localization and Monitoring of Smart Energy Meters (SEM)
}

\author{
Griffith S. Klogo \\ Department of Computer \\ Engineering, KNUST, Kumasi, \\ Ghana
}

\author{
James D. Gadze \\ Department of Electrical and \\ Electronics Engineering, \\ KNUST, Kumasi, Ghana
}

\author{
Henry Diawuo \\ Department of Electrical and \\ Electronics Engineering, \\ KNUST, Kumasi, Ghana
}

\begin{abstract}
This paper evaluates the radio coverage of anchor nodes and reliability in an anchor-based wireless sensor network platform used in locating and monitoring smart energy meters. Knowledge of the location of smart energy meters is critical for network functionalities such as monitoring, billing and energy budget planning. One efficient technique of determining the location of field monitoring and measuring devices such as smart energy meters is the anchor-based localization. Anchor-based localization is a technique in which the unknown node's location is determined with reference to a location aware node. Anchor-based localization techniques require anchor nodes having good radio coverage to the unknown nodes. The error associated with localization is high when anchor nodes are not optimally accessed by unknown nodes in the network. This paper performed a radio coverage analysis and evaluated the reliability of anchor nodes within a network perimeter for the localization and monitoring of smart energy meters. An outage probability was used to verify reliability of the network for the localization and monitoring of the smart energy meters. Our result show that for a 100 by 100 meter perimeter, four anchor nodes will provide good reliability of radio coverage for the localization and monitoring of smart energy meters.
\end{abstract}

\section{Keywords}

Radio coverage, localization, monitoring, smart energy meters, anchor-based localization.

\section{INTRODUCTION}

Unlike traditional energy meters, smart energy meters have wireless communication, sensory, actuating and signal processing capabilities. These smart energy meters can form a network wireless sensors performing automated metering, demand side management, billing and disconnecting defaulting users remotely. Following the example of many technological developments, Wireless Sensor Networks (WSNs) were a concentrated field of application for military purpose. Nowadays, smart environments are deployed everywhere, and sensor networks can be used in many different scenarios. Wireless sensor networks consist of dispersed independent sensors that monitor physical properties in an environment. The advancement in modern technologies (Micro-Electro-Mechanical Systems (MEMS) and VLSI technology) in manufacturing sensor nodes has led to their large scale deployment for numerous applications. Wireless sensor networks are particularly motivating in unsafe or remote environments, or when a large number of sensor nodes have to be deployed. Smart energy metering is a typical application area of wireless sensor networks. Locating and monitoring power consumption of smart energy meters remotely helps in the reduction of operational and commercial losses of utility providers. Remote localization and monitoring of smart energy meters does provide a means to detect fraud, illegal connections and tampering of energy meters in good time. Localization and monitoring of smart energy meters will provide an alternative means of energy budget planning and billing purposes. With the huge number of nodes deployed, locating and monitoring smart energy meters is usually a challenge in wireless sensor network. Figure 1 shows the proposed clustered network of smart energy meters for locating and monitoring power consumption of users and other network functions. Localization is the process of determining the positional coordinates of sensor nodes whose location is not known apriori [1] [2] [3]. Localization schemes are classified on the basis of the strategies employed in determining the location of unknown sensor nodes. Localization process that estimates the positional coordinates of unknown sensor nodes (static or mobile) relative to other sensor nodes with known location is referred to as an Anchorbased localization [1]. Anchor nodes are sensor nodes in the network that have their position known. Localization schemes are referred to as Anchor-Based or Anchor free if the technique involves estimating the location of the unknown sensor node in reference to an anchor node or without reference to an anchor node respectively [4] [5]. Anchor nodes as shown in figure 1 , are those nodes in the network that know their location by means of direct deployment at known coordinates, hard coding the location into the hardware or acquired by using locational systems such as a Global Positioning System (GPS) receiver [1] [6-9]. The anchor nodes in figure 1 acts as both cluster heads and reference nodes with which all other nodes determine their location. Setting up anchor nodes is both labour and hardware intensive, hence the need for localization techniques that will require few anchor nodes without compromising accuracy. Most researchers have established that anchors are best when placed uniformly on the perimeter of the network as shown in figure 1 [6] [8] [9]. The position of anchor nodes in a network does affect the accuracy of localization, hence the need for optimal placement strategies. Even though research has proposed perimeter based placement, it is evident that the position or placement of anchor nodes does depend on the application field of the sensor network [5-6] [9-10]. Optimally placed anchor nodes will ensure quality radio coverage of the network perimeter for the localization and monitoring of unknown nodes. Using few anchor nodes without compromising localization accuracy requires that unknown nodes have quality radio coverage from the anchor nodes. Radio coverage quality of the anchor nodes for the localization of smart energy meters in WSNs has a fundamental impact on the network performance and affects as well the reliability of monitoring the smart energy meters. Considering the circled region in figure 1 which is a 
100 by 100 meter perimeter, we evaluate the radio coverage of the anchor nodes for the localization and monitoring of the smart energy meters within the network.

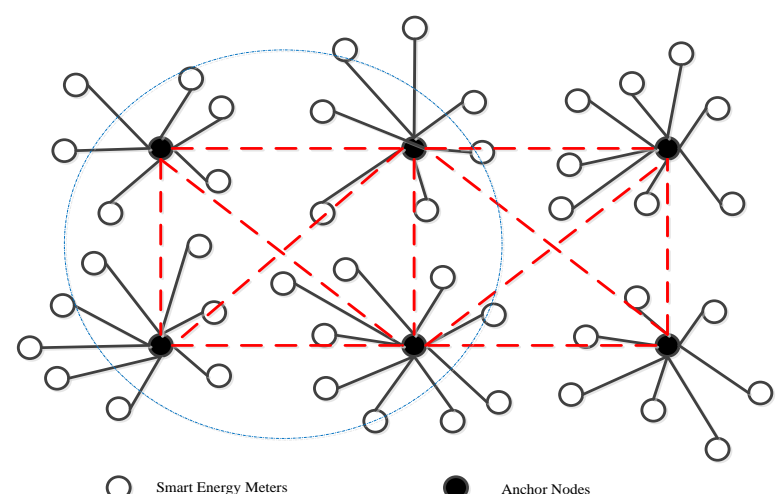

Fig 1: Proposed clustered network of smart energy meters

\section{RADIO COVERAGE EVALUATION}

The propagation and coverage of radio signals is affected by several factors contributing to the degradation of its quality. The effects of these factors are even more substantial on the propagation of wireless signals from sensor nodes with lowpower radios, typically used in wireless sensor networks. Consequently, radio links in WSNs are often unpredictable in various environments. Due to the unplanned nature of most settlements in the Africa sub-region, radio communication of smart energy meter does suffer signal loss. In wireless communication, reduction of radio signal strength in settlement environments can be with the combined path loss and shadowing model in equation 1 . Radio coverage is the expected percentage of scenes within the wireless sensor network where the received power across the network is above a given minimum. The radio coverage evaluation performed in this paper assumed the combined path loss and shadowing model in equation 1 . With this model, average $\mathrm{dB}$ path loss is described by the path loss model while shadow fading, with a mean of $0 \mathrm{~dB}$, creates variations about the pathloss as shown in figure 2 . The path loss obeys the simplified path-loss model with $\mathrm{K}=-35.54 \mathrm{~dB}$ and the path-loss exponent $\gamma$ and the shadowing obeys the Gaussian log-normal model $(\psi d B)$ with mean given by the path-loss model and standard deviation $\sigma_{\psi d B}[11]$.

$$
\begin{gathered}
\frac{P_{r}}{P_{t}} d B=10 \log _{10} K-10 \gamma \log _{10} \frac{d}{d_{O}}-\psi_{d B} \\
\text { Where, } \psi_{d B}=10 \log _{10} \psi \text { and } \psi=P_{t} / P_{r}
\end{gathered}
$$

where

\section{Transmitted power $\left(P_{t}\right)$}

Received power $\left(P_{r}\right)$

Distance to receiver $(d)$

Reference distance $\left(d_{o}\right)$

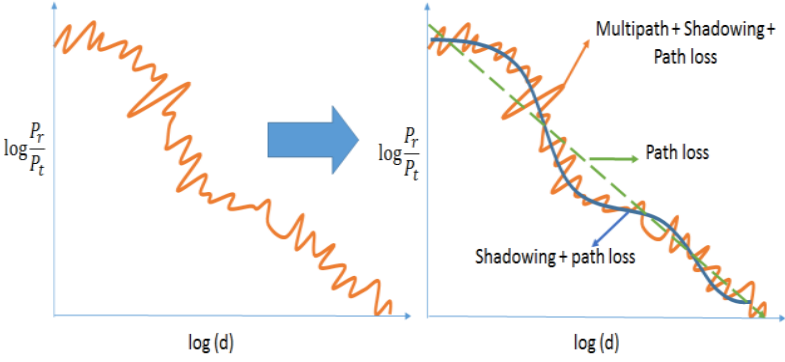

Fig. 2: Graph of path loss, shadowing and multipath fading versus distance [12]

\subsection{Outage Probability of Anchor Nodes}

The combined effects of the path loss and shadowing does affect the radio coverage of anchor nodes. This also affects the localization and monitoring of the smart energy meters in the network. Random fluctuation in the quality of radio signal can be attributed to the shadow fading and noise in the sensor network. The shadowing phenomenon does give rise to random variation of the received power levels of unknown nodes at various distances within the sensor network. The Outage Probability as in equation 2 is used as a measure of the degree to which the transmitted signal levels from the anchor nodes within the sensor network will fall below the minimum power level of the sensor nodes receiver's sensitivity. In other words, the outage probability is the measure of the connectivity between the known sensor node and the anchor nodes within the network. An outage probability of $1 \%$ is most desirable in wireless systems. This research used the outage probability to estimate the failure of anchor nodes in the localization process.

$$
p\left(P_{r}(d) \leq P_{\min }\right)=1-Q\left(\frac{P_{\min }-\left(P_{t}+10 \log _{10} K-10 \gamma \log _{10}\left(d / d_{o}\right)\right)}{\sigma_{\psi_{d B}}}\right) 2
$$

\subsection{Simulation setup}

The simulation environment was setup in a square perimeter network of $100 \times 100$ meters as shown in figure 3. Anchor nodes are placed in the four corners of the network [6]. Anchor nodes have same specification as all other nodes in the network but have the advantage of known location as shown in table 1. The simulation is carried out in four different environments; line of sight, sub-urban, residential and non-line of sight. The combined path loss and shadowing model is used in all of the environments to evaluate the radio coverage in the network using the MATLAB simulation tool.

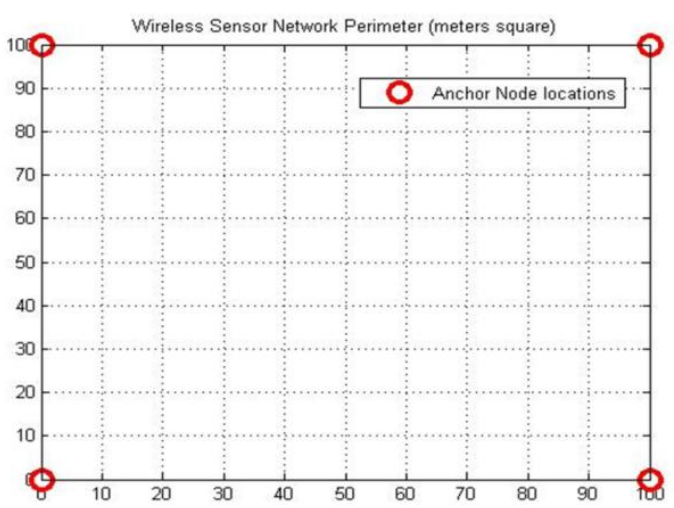

Fig 3: Anchor Nodes in a square perimeter of $100 \times 100$ meters 


\subsection{Simulation Parameters}

Table 1 shows a list of specifications used in the simulation of the radio coverage of the anchor nodes in the network. Both sensor nodes and anchor nodes have the same radio module specification of a typical MICAz sensor node. Table 2 shows the simulation parameters.

Table 1: Typical Specification of a sensor node (MICAz) [13]

\begin{tabular}{|l|l|}
\hline \multicolumn{2}{|l|}{ RF Transceiver } \\
\hline Frequency band & $2400 \mathrm{MHz}$ to $2483.5 \mathrm{MHz}$ \\
\hline Transmit (TX) data rate & $250 \mathrm{kbps}$ \\
\hline RF power & $-24 \mathrm{dBm}$ to $0 \mathrm{dBm}$ \\
\hline Receive Sensitivity & $-90 \mathrm{dBm}$ (min), $-94 \mathrm{dBm}$ (Typ) \\
\hline Outdoor Range & $75 \mathrm{~m}$ to $100 \mathrm{~m}$ \\
\hline Indoor Range & $20 \mathrm{~m}$ to $30 \mathrm{~m}$ \\
\hline
\end{tabular}

\subsection{Simulation Results}
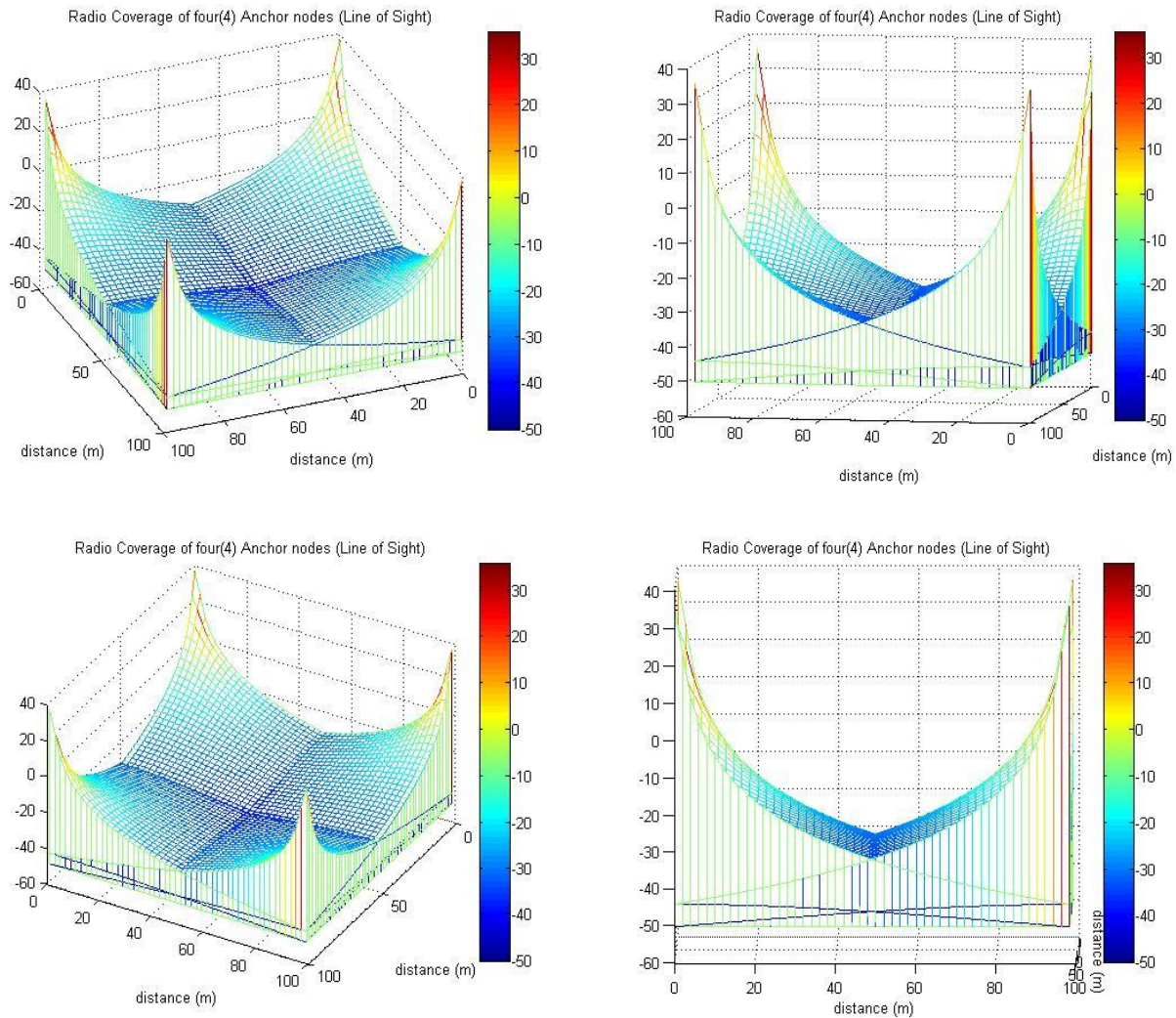

Fig 4: Radio Coverage of four (4) anchor nodes in Line of Sight

\begin{tabular}{|c|c|}
\hline Frequency Band & $2400 \mathrm{MHz}$ to $2483.5 \mathrm{MHz}$ \\
\hline RF Power & $\mathrm{OdBm}$ \\
\hline Receiver Sensitivity & $-90 \mathrm{dBm}(\min ),-94 \mathrm{dBm}(\max )$ \\
\hline Tx and Rx Gain (dB) & $1 \mathrm{~dB}$ \\
\hline Maximum distance (d) & $100 \mathrm{~m}$ \\
\hline Reference distance (do) & $1 \mathrm{~m}$ \\
\hline Path loss Exponent (n) [14] & $\begin{array}{ll}2 & \text { (Line of Sight) } \\
2.8 & \text { (Sub-urban area) } \\
2.93 & \text { (Residential area) } \\
3.5 & \text { (Non line of sight) }\end{array}$ \\
\hline $\begin{array}{l}\text { Gaussian random variable with standard } \\
\text { deviation (sigma) [14] }\end{array}$ & $\begin{array}{ll}6 \mathrm{~dB} & \text { (Line of Sight) } \\
9.6 \mathrm{~dB} & \text { (Sub-urban area) } \\
7.85 \mathrm{~dB} & \text { (Residential area) } \\
11.7 \mathrm{~dB} & \text { (Non line of sight) }\end{array}$ \\
\hline Gaussian random variable mean & 0 \\
\hline
\end{tabular}



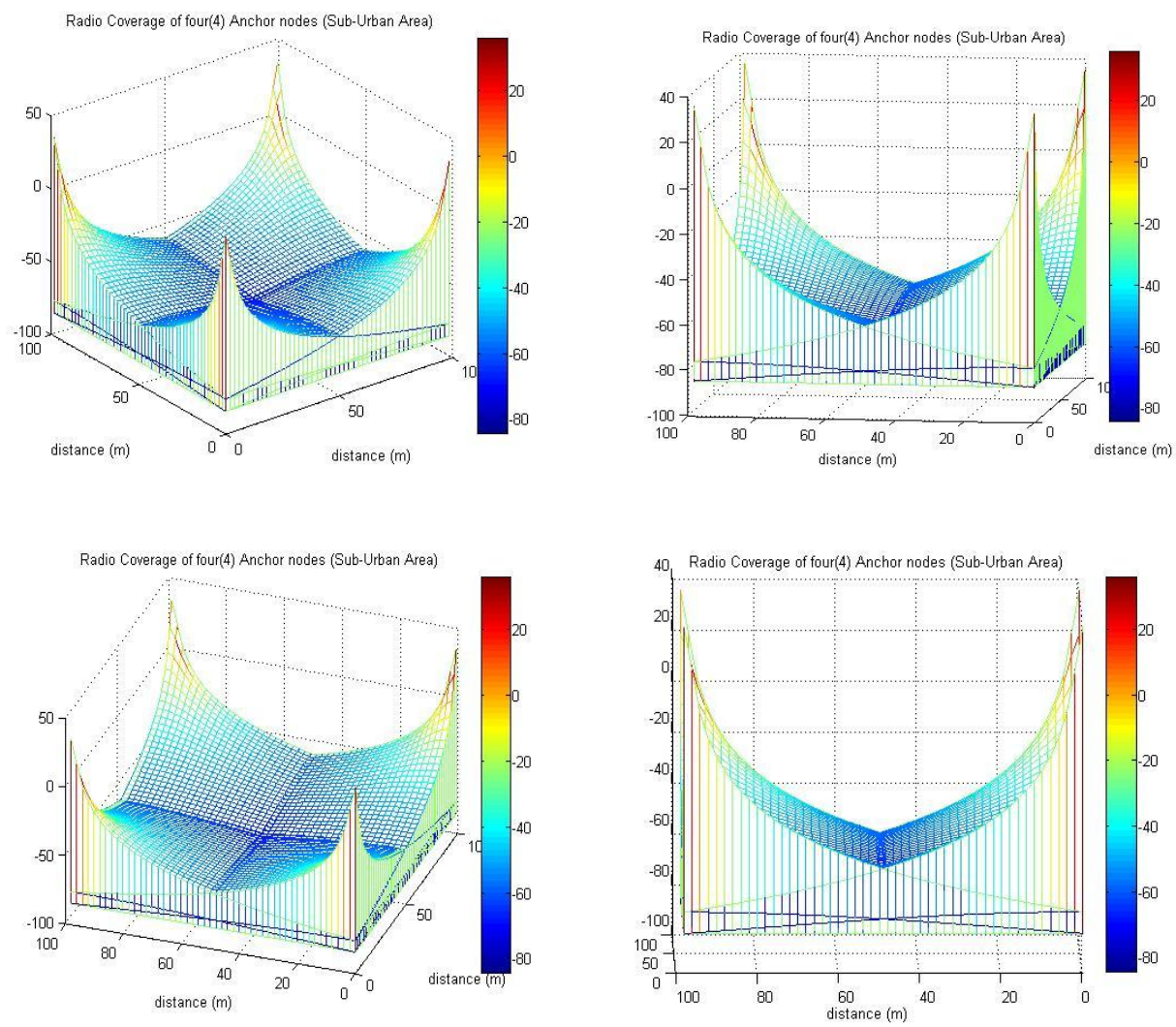

Fig 5: Radio Coverage of four (4) anchor nodes in Sub-urban Environment
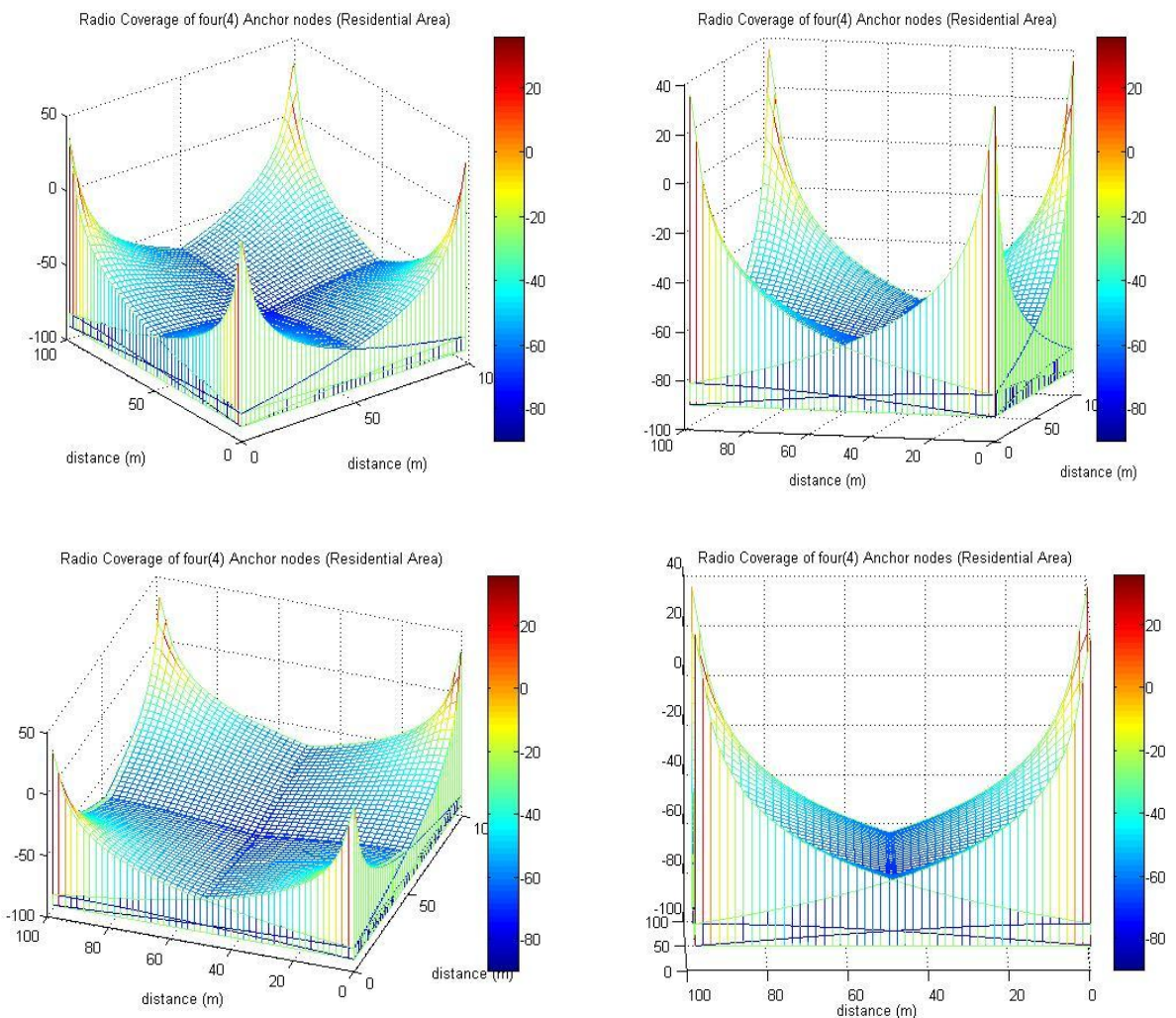

Fig 6: Radio Coverage of four (4) anchor nodes in Residential Environment 

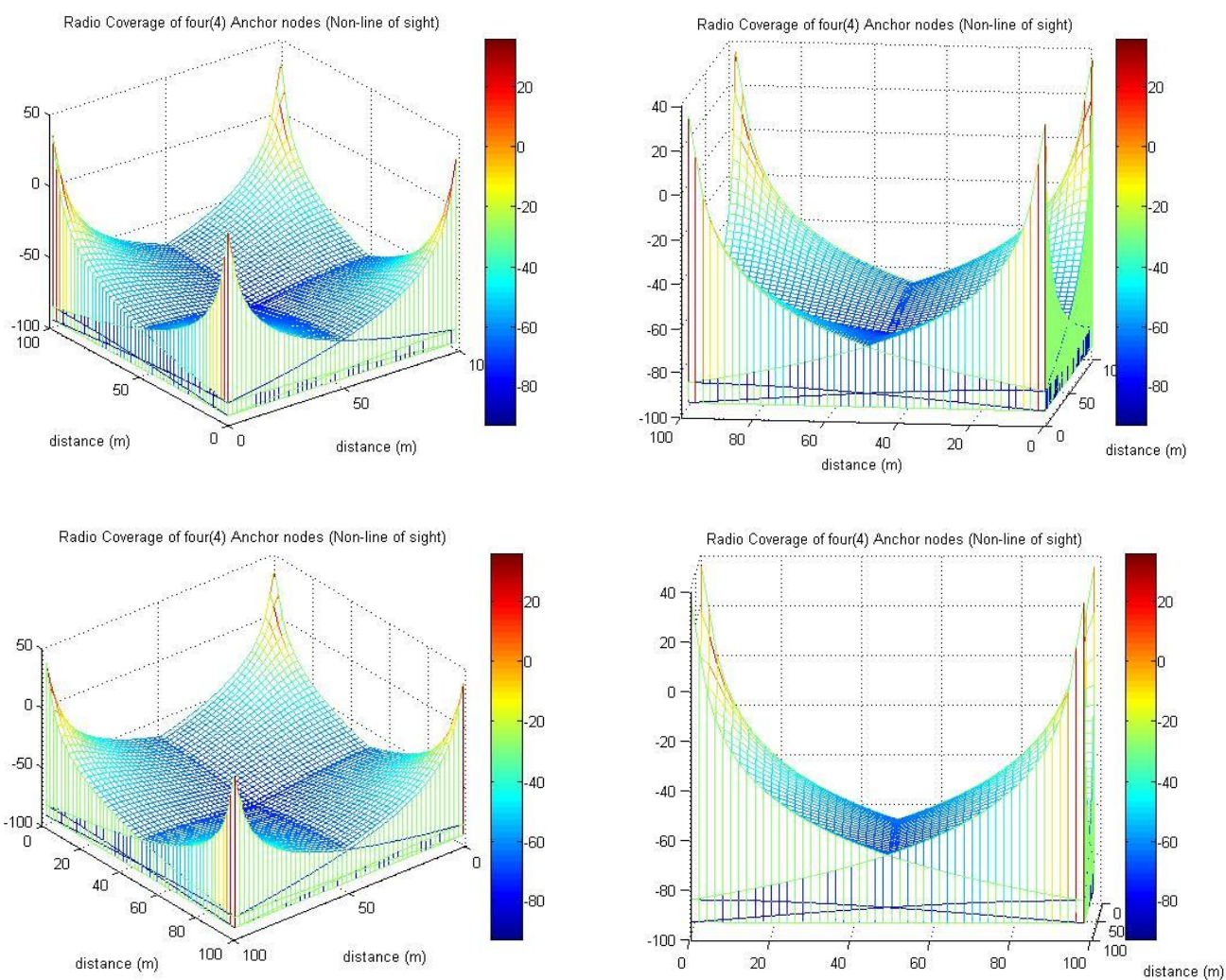

Fig 7: Radio Coverage of four (4) anchor nodes in Non-Line of Sight

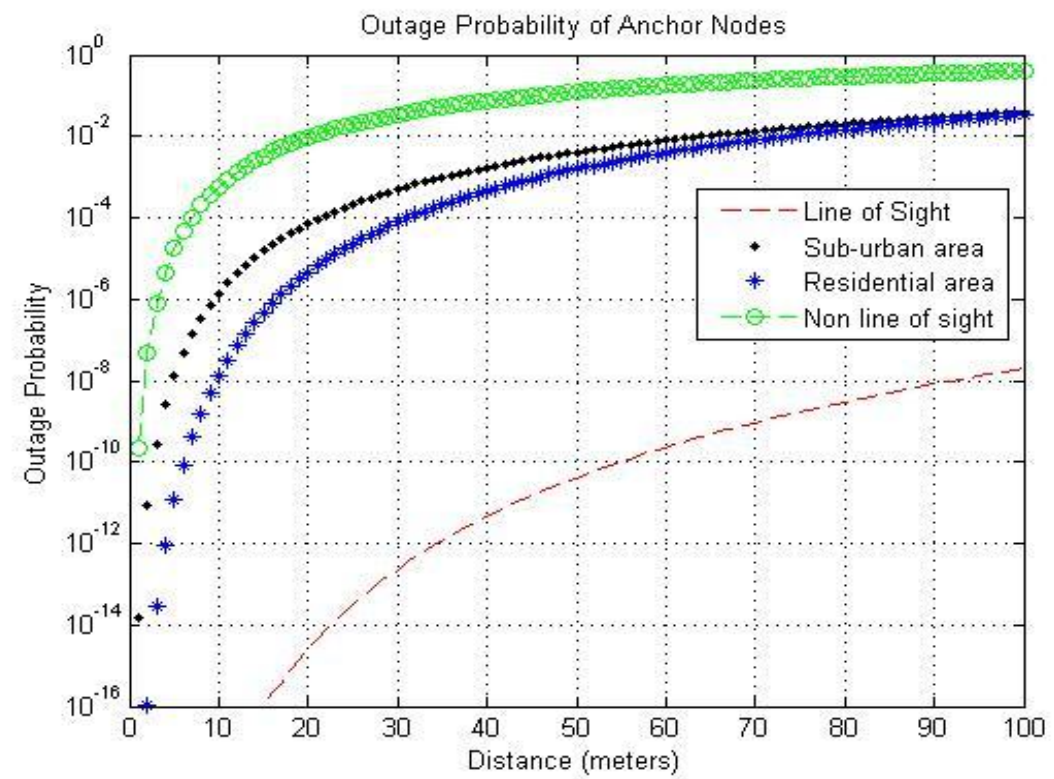

Fig 8: Outage Probability of Anchor nodes in different Environments

\section{DISCUSSION OF RESULTS}

Radio coverage of anchor nodes plays an important role in wireless sensor network localization and monitoring. Locating and monitoring smart energy meters will require quality radio signals to the meters in the network from the anchor nodes or concentrators. Using the radio module specifications of a typical sensor node, this paper performed a measure of radio/signal coverage to all smart energy meters within a network. The radio coverage of anchor nodes placed on the perimeter of 100x100 meter network is performed for four environmental conditions. This paper performed the simulation in the environmental conditions of line of sight, sub-urban, residential and non-line of sight. The signal strength in all of these environments is compared to the radio module specification in table 1 . The receiver sensitivity is the minimum power level (in $\mathrm{dBm}$ or $\mathrm{mW}$ ) that can be detected 
by the sensor nodes (smart energy meters) for the receiver to accurately decode a given signal. Sensitivity is purely a receiver specification and is independent of the transmitter in this case the anchor nodes. The results of figure 4, clear illustrate the quality radio coverage in line of sight environment. The radio signal strength (RSS) is found to be higher than the typical and minimum receiver sensitivity specified in the radio module specification of a typical wireless sensor node in table 1 . In four different views of the network, figure 4 shows how the network can be fully covered by the anchor nodes for localization and monitoring with minimum power level of $-50 \mathrm{dBm}$. Figure 5 shows the results from the simulation in the sub-urban environment. The network is fully covered in this environment with minimum power level of $-80 \mathrm{dBm}$ which is less than the sensitivity of the receivers on the smart energy meters. The simulation result for residential environment is shown in figure 6 and that of non-line of sight environment is shown in figure 7. The minimum power level for both residential and non-line of sight conditions is $-80 \mathrm{dBm}$ which is below the typical receiver sensitivity level. Outage probability is performed for all the anchor nodes at various distances to the receivers (smart energy meters). Figure 8 shows the results of the outage probability for the various environments considered in this paper. An outage probability of about $1 \%$ is usually allowed in wireless networks. From figure 8 , it is evident that the probability of outage is very low within the network for line of sight, sub-urban, and residential environments. Outage probability in non-line of sight is a little more than $1 \%$ but the presence of multiple anchors does reduce the probability of outage within the network.

\subsection{Conclusion}

The quality of radio coverage of anchor nodes for the localization and monitoring of smart energy meters does affect the accuracy of localization and reliable monitoring. In this paper, simulation of the radio coverage and outage probability of anchor nodes was performed to assess the quality of radio signals that will be received by smart energy meter. The results using typical specifications of radio module of a sensor nodes, shows that smart energy meters with the specification in table 1 can be located and monitored with the anchor nodes having the same specification on the four corners of a square perimeter of $100 \times 100$ meters.

\section{REFERENCES}

[1] G. S. Klogo and J. D. Gadze, "Energy Constraints of Localization Techniques in Wireless Sensor Networks (WSN): A Survey" International Journal of Computer Applications (0975 - 8887), Vol. 75- No.9, Aug. 2013, pp 44-52

[2] U. Nazir, M. A. Arshad, N. Shahid and S.H. Raza, "Classification of Localization Algorithms for Wireless Sensor Network: A Survey", 2012 International Conference on Open Source Systems and Technologies (ICOSST), pp 1-5
[3] N. Salman, H. K. Maheshwari, A.H. Kemp and M. Ghogho, "Effects of anchor placement on mean-CRB for localization", 2011 The 10th IFPI Annual Mediterranean Ad Hoc Networking Workshop, pp 115-118

[4] Chia-Ho Ou, Member, IEEE and Wei-Lun He, "Path Planning Algorithm for Mobile Anchor-Based Localization in Wireless Sensor Networks", IEEE Sensor Journal, Vol. 13, NO. 2, February 2013, pp 466-475

[5] S. Tian, X. Zhang, X. Wang, P. Sun and H. Zhang, "A Selective Anchor Node Localization Algorithm for Wireless Sensor Networks", 2007 International Conference on Convergence Information Technology , pp 358-362

[6] G. S. Klogo, J. D. Gadze and H. Nunoo-Mensah, "On the Number of Anchor Nodes for the Localization of Smart Energy Meters (SEM) in an African Environment", Submitted to the International Journal of Electrical Power \& Energy Systems.

[7] Y. Wang, X. Wang, D. Wang, Members, IEEE and D. P. Agrawal, Fellow, IEEE, "Range-Free Localization Using Expected Hop Progress in Wireless Sensor Networks", IEEE Transaction on Parallel and Distributed Systems, Vol. 20, NO. 10, October 2009, pp 1540-1552

[8] R. Akl, K. Pasupathy and M Haidar, "Anchor Nodes Placement for Effective Passive Localization", 2011 International Conference on Selected Topics in Mobile and Wireless Networking (ICOST), pp 127-132

[9] J. N. Ash and R. L. Moses, "On Optimal Anchor Node Placement in Sensor Localization by Optimization of Subspace Principal Angles", ICASSP @2008 IEEE, pp 2289-2292

[10] S. Tian, X. Zhang, X. Wang, P. Sun and H. Zhang, "A Selective Anchor Node Localization Algorithm for Wireless Sensor Networks", 2007 International Conference on Convergence Information Technology , pp 358-362

[11] Andrea Goldsmith, Wireless Communications, Cambridge university press, 2005

[12] Channel modeling - an Introduction, http://www.gaussianwaves.com/2013/07/channelmodeling-an-introduction/, accessed on 30th November, 2013.

[13] Micaz datasheet, Crossbow Technology, Inc., Document Part Number: 6020-0060-04 Rev A

[14] Fading and shadowing, http://morse.colorado.edu/ tlen5510/text/classwebch4.ht $\mathrm{ml}$, accessed on 18th September 2013. 